\title{
Robotic single-site surgery versus laparo-endoscopic single-site surgery in ovarian cystectomy: A retrospec- tive analysis in single institution
}

\author{
Seongmin Kim, Kyung Jin Min, Sanghoon Lee, Jin Hwa Hong, Jae Yun Song, Jae Kwan Lee, Nak Woo Lee \\ Department of Obstetrics and Gynecology, Korea University College of Medicine, Seoul, Korea
}

Objective: Robotic single-site (RSS) surgery is being utilized in gynecologic surgery. However, its cost-effectiveness and limitations of the system make the application of the technique to benign surgery controversial. We compared the surgical outcome and learning curve of RSS to those of laparoendoscopic single-site (LESS) surgery.

Methods: Patients who underwent ovarian cystectomy from January 2013 to December 2018 at Korea University Anam Hostital were divided into two groups according to the surgery: RSS or LESS. After exclusion of ineligible patients, 29 patients who underwent RSS surgery and 80 patients who underwent LESS surgery were included in this study. Surgical outcome data regarding bleeding, surgical time, length of stay, and the learning curves of surgical time were obtained using the cumulative sum method.

Results: Patient demographics showed no differences between the groups except for tumor histology. There were no differences in bleeding, postoperative length of stay, surgical wound complication, or surgical preparation time between the groups. RSS surgery had a longer surgical time than LESS surgery ( $130.41 \mathrm{vs.} 96.96$ minutes; $P<0.001)$. In a learning curve analysis, seven cases were required to be proficient in docking the RSS system. Comparing the learning curves of surgical time, RSS surgery required fewer cases to attain proficiency than LESS surgery (19 vs. 22 cases).

Conclusion: RSS ovarian cystectomy is a feasible and safe option with the benefit of a quick learning curve. The longer surgical time could be overcome with further improvement of surgical techniques and development of the robotic instruments.

Key Words: Ovary; Cystectomy; Laparoscopy; Learning curve

\section{INTRODUCTION}

Laparoendoscopic single-site (LESS) surgery has been in- troduced into the field of gynecologic surgery, which has the benefit of minimal scarring [1-3]. Beyond overcoming surgical limitations in angulation and device manipulation,

\footnotetext{
- Received: April 13, 2019 • Revised: October 18, 2019 • Accepted: October 21, 2019

- Correspondening author: Jae Yun Song

Department of Obstetrics and Gynecology, Korea University Medical Center, 73 Inchon-ro, Seongbuk-gu, Seoul 02841, Korea

E-mail: sjyuni105@gmail.com
}

This is an Open Access article distributed under the terms of the Creative Commons Attribution Non-Commercial License (http://creativecommons.org/ licenses/by-nc/4.0) which permits unrestricted non-commercial use, distribution, and reproduction in any medium, provided the original work is properly cited. 
this technique has been applied even in surgeries with a high degree of difficulty [4]. However, surgeons need a prolonged learning curve period to achieve a proficiency without technical difficulties. Among diverse laparoendoscopic surgical techniques, intracorporeal suturing of ovarian tissue after ovarian cystectomy is one of the most difficult procedures and it is difficult to become skilled the technique in a short period.

The robotic surgical technology has improved visualization and surgical dexterity, and surgeons can try the surgical techniques of laparotomy in minimally invasive surgery due to the ergonomic layout. Though robotic single-site (RSS) surgery may not be utilized as much as multiport robotic surgery, it still has benefits in terms of intracorporeal suturing, avoiding crush by surgical instruments, and the materialization of triangulation compared to LESS surgery [5]. Further, patients with benign ovarian tumors including mature cystic teratoma, cystadenoma, or endometriosis, are usually in childbearing age, and they may be sensitive and have anxiety regarding surgical scars [6]. Additionally, suturing remnant ovarian capsule provides the benefit of avoiding thermal damage to ovarian tissue in preserving ovarian function. The aims of this study were to compare surgical outcomes of LESS and RSS surgeries for adnexal tumors and to investigate if RSS surgery is feasible for a patient with a benign adnexal mass.

\section{MATERIALS AND METHODS}

\section{Patients}

This retrospective study evaluated 125 patients with benign ovarian tumor lesions who underwent ovarian cystectomy using either LESS or RSS in our institution (Fig. 1). There were 16 ineligible patients because of the use of an additional port or a combined surgery. Finally, 29 patients underwent RSS and 80 patients underwent LESS from January 2013 to December 2018.

\section{Surgical techniques}

In LESS surgery, a 15-20 mm umbilical incision was made with the Hasson method. A Lapsingle ${ }^{\circledR}$ trocar (Sejong Medical, Co. Ltd., Seoul, Korea) or an Octo ${ }^{\text {TM }}$ Port trocar (DalimSurgNET Co. Ltd., Seoul, Korea) was used for instrument application. Ovarian cystectomy was defined as a surgery peeling only the cystic tumor lesions and sparing all intact ovarian tissue and the uterus. The tumors were removed by cystectomy and the contralateral ovary was preserved. We used delayed absorbable suture devices in all patients for remnant ovarian suture by an intracorporeal continuous running suture method. All resected tumors were removed using a surgical bag to avoid tissue dissemination. After surgery, the umbilical incision was closed layer by layer from the fascia to the skin.

A da Vinci Si or Xi surgical system (Intuitive Surgical Inc., Sunnyvale, CA, USA) was used for RSS surgery. This in-

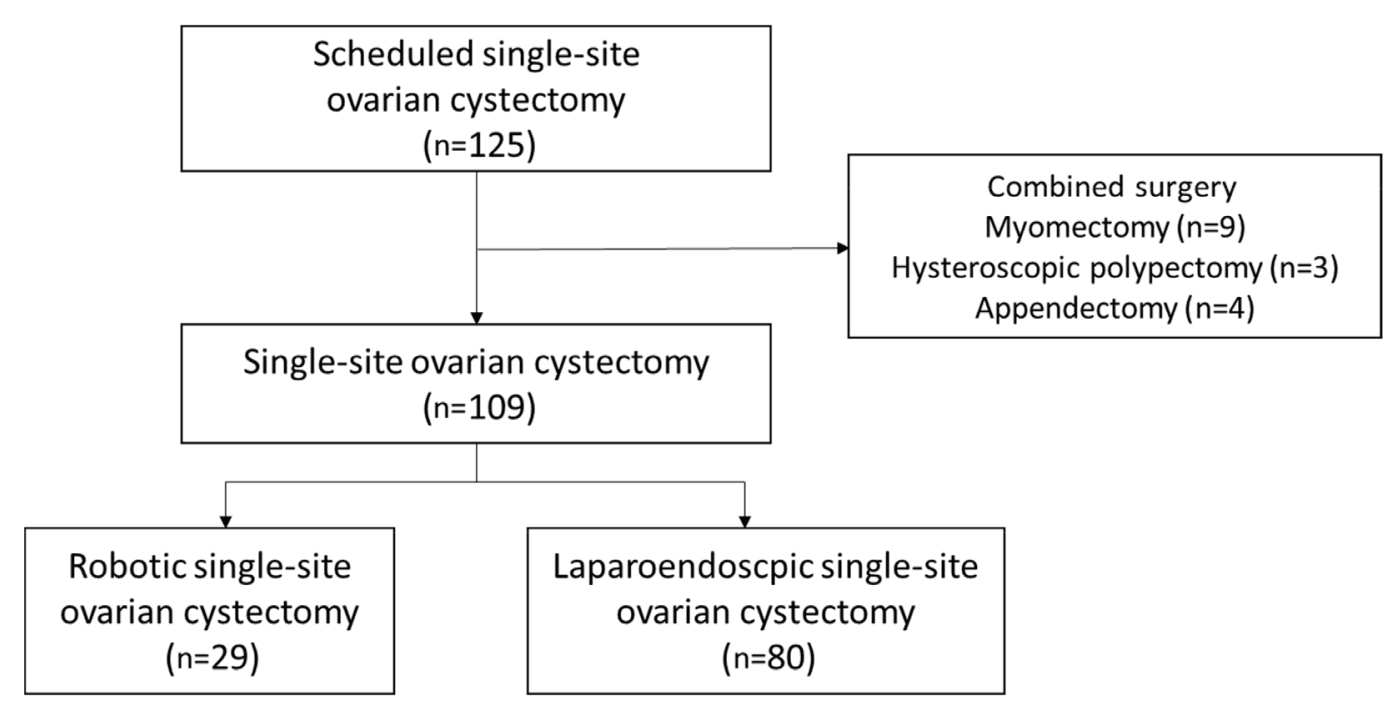

Fig. 1. Patient information. 
cluded the same surgical procedures of LESS surgery, but only a Lapsingle ${ }^{\circledR}$ trocar was used for docking the robotic arms. A central arm for the laparoscope was inserted, and then the other two arms were located. During the surgery, the surgical procedures were not different from those of LESS surgery. All resected tumors were also removed using a surgical bag. At the end of the surgery, the umbilical incision was closed in the same manner as in the LESS procedure.

\section{Statistical analysis}

Variables such as patient age, body mass index (BMI), histological test results, operative time (preparation time and operating time), estimated blood loss, days of hospitalization, and complications were analyzed under the ethical board's approval. Preparation time was defined as the time from applying anesthetic agents to incision time and surgical time was the time from first incision to the time of closure. Docking time was checked only in RSS surgery, and it was the time needed for the docking of the robotic devices.

Learning curves were analyzed by consecutive cases using the cumulative sum (CUSUM) method. A CUSUM analysis was used to quantitatively assess the learning curve of docking time and surgical time (CUSUM-DT and CUSUM-ST, respectively). This technique provides a graphical representation of the trend in the outcome of consecutive procedures performed over time since it is a plot of the cumulative total of differences between each data point and the mean of all data points. This approach gives a visual representation of the learning curve.

We used the IBM SPSS Statistics software (version 22.0; IBM Corp., Armonk, NY, USA) for the statistical analysis. A Kolmogorov-Smirnov test was used to verify the standard normal distribution assumptions. A Student's t-test and a Mann-Whitney $U$ test were used for parametric and nonparametric variables, respectively. Differences between proportions were compared using a Fisher's exact test or a chi-squared test. A level of significance was established as $P<0.05$ for all statistical tests.

\section{RESULTS}

The patient demographics are shown in Table 1. No significant statistical differences were found with respect to

\section{Table 1. Patient demographics and characteristics}

\begin{tabular}{|c|c|c|c|}
\hline Variable & RSS (n=29) & LESS $(n=80)$ & $P$-value \\
\hline Age (years) & $27.69 \pm 5.50$ & $29.34 \pm 8.65$ & 0.241 \\
\hline $\mathrm{BMI}\left(\mathrm{kg} / \mathrm{m}^{2}\right)$ & $22.51 \pm 3.94$ & $22.8 \pm 3.70$ & 0.428 \\
\hline Parity & & & 0.100 \\
\hline Virgin & $4(13.8)$ & $18(22.5)$ & \\
\hline Nulliparous & $23(79.3)$ & $46(57.5)$ & \\
\hline Multiparous & $2(6.9)$ & $16(20.0)$ & \\
\hline History of abdominal surgery & & & 0.505 \\
\hline Present & $2(6.9)$ & $9(11.3)$ & \\
\hline Absen & $27(93.1)$ & $71(88.7)$ & \\
\hline Tumor size on imaging (cm) & $7.99 \pm 3.77$ & $8.29 \pm 4.30$ & 0.709 \\
\hline Tumor location & & & 0.820 \\
\hline Right & $15(51.7)$ & $36(45.0)$ & \\
\hline Left & $11(37.9)$ & $34(42.5)$ & \\
\hline Bilateral & 3 (10.3) & $10(12.5)$ & \\
\hline Histology & & & $0.013(0.429)^{a)}$ \\
\hline Dermoid cyst & $16(55.2)$ & $30(37.5)$ & \\
\hline Endometriosis & $6(23.1)$ & $21(26.3)$ & \\
\hline Cystadenoma & $6(23.1)$ & $7(8.8)$ & \\
\hline Hemorrhagic corpus luteum & $\mathrm{O}(0)$ & $21(26.3)$ & \\
\hline Fibroma & $1(3.4)$ & $1(1.3)$ & \\
\hline
\end{tabular}

Values are presented as mean \pm standard deviation or number (\%).

RSS, robotic single-site; LESS, laparoendoscopic single-site; BMI, body mass index.

a) $P$-value inside parenthesis is comparing tumor histology of RSS to that of elective LESS only. 
age, BMI, parity, history of abdominal surgery, tumor location, and suspected tumor size on imaging including ultrasonography, computed tomography, or magnetic resonance imaging. However, tumor histology was different between the two groups because of the inclusion of emergency surgery of the hemoperitoneum due to rupture of the hemorrhagic corpus luteum or a torsion of a dermoid cyst. This difference was resolved after the exclusion of these cases.

As seen in Table 2, variables such as preparation time, surgical time, estimated blood loss, hospital stay, and decreased hemoglobin level were not different between the two groups. The preparation time of both groups were similar, but the surgical time was significantly longer in the RSS group than in the LESS group. No conversions from minimally invasive surgery to laparotomy or abdominal surgery were required in any of the 109 cases. Additional trocar insertion was necessary in two LESS cases due to severe adhesion from a previous surgical history. There were one and two umbilical hernia cases in the RSS and LESS groups, respectively. All three of these umbilical hernia cases belong to an initial period of single-site surgery in our institution and were of patients who were relatively obese. After excluding the cases of unscheduled emergent surgery, the amount of estimated blood loss was less in the LESS group, but the hemoglobin level showed no difference between the groups. The surgical time was still longer in the RSS group.

Fig. 2 shows the docking time plotted chronologically according to the case number. This learning curve was obtained by plotting the cumulative sequential differences between each data point and the average docking time, using
CUSUM, which was best modeled as a third-order parabola (CUSUM-DT). It showed a main slope change after the 7th case, making an initial positive slope followed by a negative slope. We also compared the learning curves of surgical time for the two groups (CUSUM-ST). In RSS, a triphasic curve was most suitable for CUSUM-ST, which showed a main slope change after 19 cases (Fig. 3A). A similar pattern was observed for the first 30 cases of LESS, but the slope changed after 22 cases (Fig. 3B).

\section{DISCUSSION}

For the last decades, the introduction of robotics in gynecological operations has been one of the most remarkable advances in the field of minimally invasive gynecologic sur-

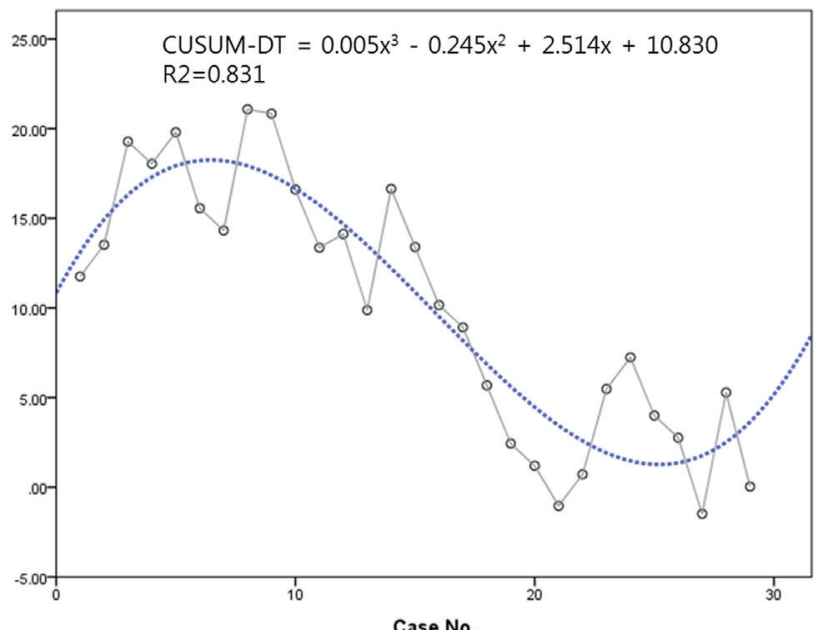

Fig. 2. Learning curve for docking time in robotic single-site surgery. CUSUM-DT, cumulative sum of docking time.

Table 2. Surgical outcomes of single-site ovarian cystectomy

\begin{tabular}{lccccc}
\hline & RSS ( $\mathbf{n = 2 9 )}$ & Total LESS ( $\mathbf{n = 8 0 )}$ & $\boldsymbol{P}_{\text {-value }}$ a) & Elective LESS ( $\mathbf{n = 5 9 )}$ & $\boldsymbol{P}$-value $^{\text {b) }}$ \\
\hline Estimated blood loss (mL) & $110.34 \pm 111.31$ & $80.49 \pm 102.16$ & 0.211 & $54.19 \pm 86.01$ & 0.019 \\
Transfusion, yes & $1(3.4)$ & $4(5.0)$ & 0.732 & $1(1.7)$ & 0.604 \\
Hemoglobin-drop & $1.60 \pm 1.10$ & $1.65 \pm 0.93$ & 0.812 & $1.74 \pm 0.92$ & 0.523 \\
Length of postoperative stay (days) & $2.55 \pm 0.99$ & $2.72 \pm 0.98$ & 0.446 & $2.67 \pm 0.71$ & 0.269 \\
Preparation time (minutes) & $24.24 \pm 5.85$ & $25.57 \pm 6.31$ & 0.351 & $25.05 \pm 6.02$ & 0.761 \\
Surgical time (minutes) & $130.41 \pm 49.59$ & $96.96 \pm 46.23$ & $<0.001$ & $109.31 \pm 39.26$ & 0.009 \\
Docking time (minutes) & $8.24 \pm 4.31$ & - & $\mathrm{N} / \mathrm{A}$ & - & $\mathrm{N} / \mathrm{A}$ \\
Additional trocar insertion & $0(0)$ & $2(2.5)$ & 0.390 & $2(3.4)$ & 0.316 \\
Umbilical hernia, yes & $1(3.4)$ & $2(2.5)$ & 0.789 & $2(3.4)$ & 0.989 \\
\hline
\end{tabular}

Values are presented as mean \pm standard deviation or number (\%).

RSS, robotic single-site; LESS, laparoendoscopic single-site; N/A, not available.

${ }^{\text {a) }} P$-value comparing RSS to total LESS; ${ }^{\text {b) }} P$-value comparing RSS to elective LESS only. 

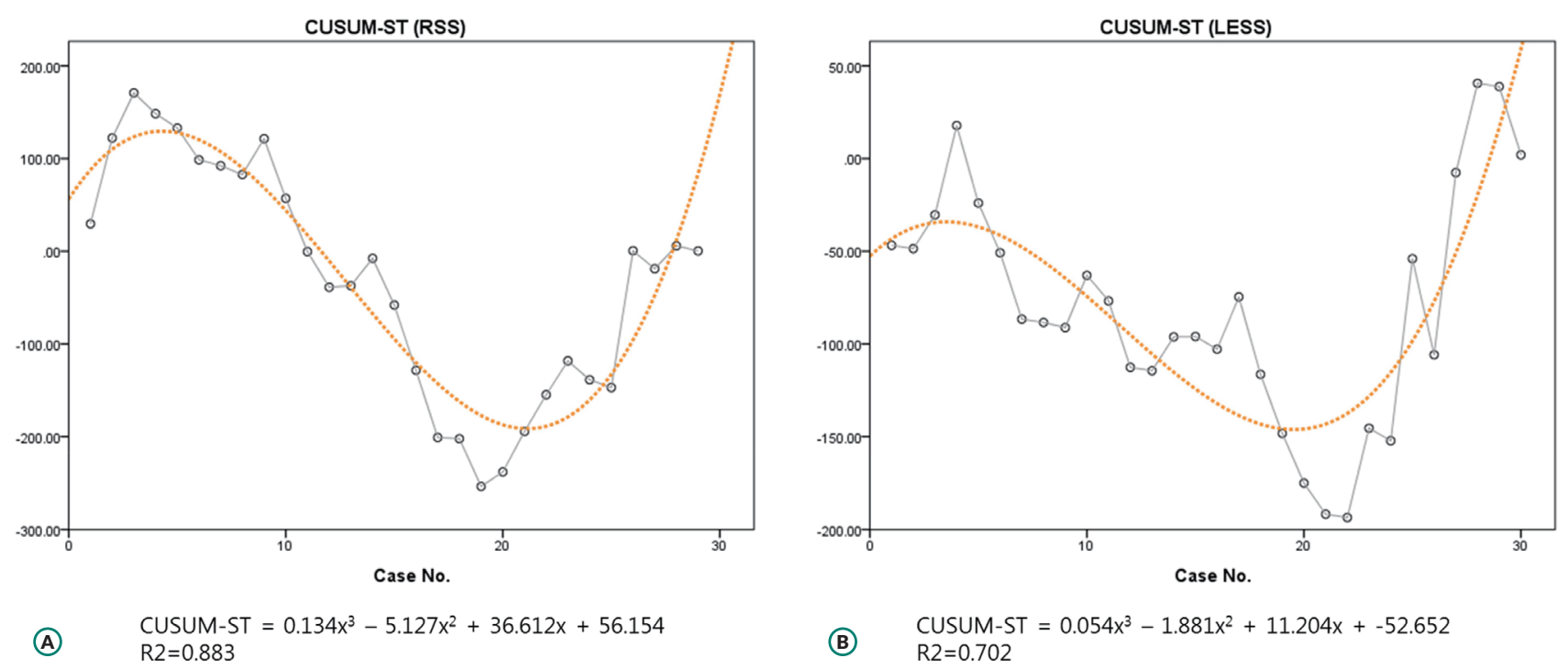

Fig. 3. Learning curves for surgical time in single-site surgery. (A) Robotic single-site surgery. (B) Laparoendoscopic single-site surgery. CUSUM-ST, cumulative sum of surgical time; RSS, robotic single-site; LESS, laparoendoscopic single-site.

gery [7]. In Korea, there is a clear trend of increasing use of robotic systems not only in oncologic surgery, but also in benign surgery [8]. RSS surgery is the most advanced technique in the field of robotic surgery, but its application is limited to selective patients due to the lack of national insurance coverage for robotic surgery in South Korea [9]. In addition, many surgeons feel uncomfortable about using the RSS system compared to the multiport robotic system and complain about the limited range of movement, insufficient strength of the robotic arms, and lack of articulation. However, single-site surgery has its own benefits such as an invisible or less scarring surgical incision, and it is reasonable when compared to LESS surgery rather than multiport robotic surgery [10].

Our data shows comparable surgical outcomes for RSS ovarian cystectomy. In our institution, there is no absolute indication of minimally invasive surgery conducted via either robotic or laparoscopic method. Surgeons decide which patients are suitable for robotic surgery and give them information about robotic surgery. Single-site surgery is usually performed on younger patients who are relatively sensitive about surgical wounds and scarring. In this study, RSS showed similar levels of blood loss, transfusion rates, lengths of stay, and wound complication rates such as hernia. However, RSS surgery had a longer surgical time than LESS surgery. After exclusion of emergency surgeries, the amount of estimated blood loss (EBL) in the operative record was larger in the RSS group. However, the rates of transfusion and hemoglobin levels showed no difference between the two groups. The EBL in the medical record could be inaccurate or omitted in certain circumstances. The surgical time was still longer in RSS surgery than in elective LESS surgery. Paek et al. [11] reported a similar outcome in an analysis of single-site surgeries of adnexal tumors. This is inevitable due to the necessary time for docking the robotic system to the patients. This does not only mean the time for initial docking, but also includes the time for changing robotic instruments, cleaning the camera, undocking, scrubbing, and donning the surgical clothes for the surgeon. Additionally, there is also a risk of error with robotic instruments. It is not surprising that RSS surgery had a longer surgical time considering these aspects.

In an analysis of the learning curve, the docking in RSS surgery did not require many cases to attain sufficient skills. In our study, the surgeon was adept at docking in RSS surgery after only seven cases. This was even faster than CUSUM-DT of multiport robotic surgery in radical hysterectomy previously reported from our institution [12]. The initial docking would be complicated because of the paradoxical positioning of the robotic arms, but once it reaches a proficiency, it is easier to dock because it does not require interport distance or consideration of the diversity of each pa- 
tient's abdominal extent [13]. In CUSUM-ST, RSS surgery required fewer cases to attain proficiency than LESS surgery. This is a definite benefit of the robotic system [14] and many surgeons could be familiar with single-site surgery using a robotic system even if they felt somewhat uncomfortable when performing LESS surgery.

This study has several limitations. First, this cohort represents a small window to the learning curve data of a single surgeon, which may not be generalizable to others. However, the single surgeon experience is important to consider, when taking into account that this surgeon has the widest experience of diverse gynecologic surgery in our country. Second, the patient data were obtained retrospectively, and selection bias could impact the results. However, the patient demographics were not different between the two groups, and we also conducted the evaluation excluding emergency surgeries for a more objective comparison.

In conclusion, the surgical outcomes of RSS ovarian cystectomy were comparable to those of LESS ovarian cystectomy. RSS ovarian cystectomy could be performed safely without complications and the RSS system requires fewer cases to gain proficiency in the learning curve of surgical time in initial cases. The long surgical time may be improved by further experience and technical refinements.

\section{Conflict of interest}

No potential conflict of interest relevant to this article was reported.

\section{References}

1. Fanfani F, Monterossi G, Fagotti A, Scambia G. Laparoendoscopic single-site hysterectomy: is it safe and feasible? Curr Opin Obstet Gynecol 2014;26:275-80.

2. Lee IO, Yoon JW, Chung D, Yim GW, Nam EJ, Kim S, et al. A comparison of clinical and surgical outcomes between laparo- endoscopic single-site surgery and traditional multiport laparoscopic surgery for adnexal tumors. Obstet Gynecol Sci 2014;57:386-92.

3. Eom JM, Choi JS, Choi WJ, Kim YH, Lee JH. Does single-port laparoscopic surgery reduce postoperative pain in women with benign gynecologic disease? J Laparoendosc Adv Surg Tech A 2013;23:999-1005.

4. Bandera CA, Magrina JF. Robotic surgery in gynecologic oncology. Curr Opin Obstet Gynecol 2009;21:25-30.

5. Kaouk JH, Goel RK, Haber GP, Crouzet S, Stein RJ. Robotic single-port transumbilical surgery in humans: initial report. BJU Int 2009;103:366-9.

6. Autorino R, Kaouk JH, Stolzenburg JU, Gill IS, Mottrie A, Tewari A, et al. Current status and future directions of robotic single-site surgery: a systematic review. Eur Urol 2013;63:26680.

7. Wright JD, Ananth CV, Lewin SN, Burke WM, Lu YS, Neugut Al, et al. Robotically assisted vs laparoscopic hysterectomy among women with benign gynecologic disease. JAMA 2013;309:689-98.

8. Yim GW, Kim YT. Robotic surgery in gynecologic cancer. Curr Opin Obstet Gynecol 2012;24:14-23.

9. Choi EJ, Rho AM, Lee SR, Jeong K, Moon HS. Robotic singlesite myomectomy: clinical analysis of 61 consecutive cases. J Minim Invasive Gynecol 2017;24:632-9.

10. Nelson RJ, Chavali JSS, Yerram N, Babbar P, Kaouk JH. Current status of robotic single-port surgery. Urol Ann 2017;9:217-22.

11. Paek J, Lee JD, Kong TW, Chang SJ, Ryu HS. Robotic single-site versus laparo-endoscopic single-site surgery for adnexal tumours: a propensity score-matching analysis. Int J Med Robot 2016;12:694-700.

12. Heo YJ, Kim S, Min KJ, Lee S, Hong JH, Lee JK, et al. The comparison of surgical outcomes and learning curves of radical hysterectomy by laparoscopy and robotic system for cervical cancer: an experience of a single surgeon. Obstet Gynecol Sci 2018;61:468-76.

13. Cela V, Freschi L, Simi G, Ruggiero M, Tana R, Pluchino N. Robotic single-site hysterectomy: feasibility, learning curve and surgical outcome. Surg Endosc 2013;27:2638-43.

14. Yohannes P, Rotariu P, Pinto P, Smith AD, Lee BR. Comparison of robotic versus laparoscopic skills: is there a difference in the learning curve? Urology 2002;60:39-45; discussion 45. 